\title{
Voltage Balancing of Multiphase FCML Converters with Coupled Inductors
}

This paper was downloaded from TechRxiv (https://www.techrxiv.org).

\section{LICENSE}

CC BY 4.0

SUBMISSION DATE / POSTED DATE

$21-07-2021 / 25-07-2021$

CITATION

Zhou, Daniel H.; Chen, Minjie (2021): Voltage Balancing of Multiphase FCML Converters with Coupled Inductors. TechRxiv. Preprint. https://doi.org/10.36227/techrxiv.15029055.v1

$\mathrm{DOI}$

10.36227/techrxiv.15029055.v1 


\title{
Voltage Balancing of Multiphase FCML Converters with Coupled Inductors
}

\author{
Daniel H. Zhou, Student Member, IEEE, Minjie Chen, Senior Member, IEEE
}

\begin{abstract}
Flying capacitor voltage balancing is critical for the performance of flying capacitor multilevel (FCML) converters. This paper investigates the intrinsic capacitor voltage balancing of multiphase FCML converters with coupled inductors. It is shown that the coupled inductor provides flying capacitor voltage balancing that minimizes steady-state imbalances due to periodic disturbances compared to converters with uncoupled inductors. A dynamic model of natural balancing of the converter is derived and used to estimate the time required for the flying capacitors to settle from an initial imbalance. The theoretical predictions are verified with analytical derivations, SPICE simulations, and experimental results. Extended derivations and experiments will be presented in the final paper.
\end{abstract}

\section{Introduction}

Many power electronics applications such as envelope tracking require dc-dc converters with low inductor current ripple and fast transient response [1]. Inductor current ripple must be minimized to improve efficiency, while bandwidth must be high to quickly respond to load transients. Figure 1 shows a two-phase, three-level FCML converter combining multiphase interleaving, multilevel switching, and coupled inductors to reduce current ripple and improve bandwidth [2-4].

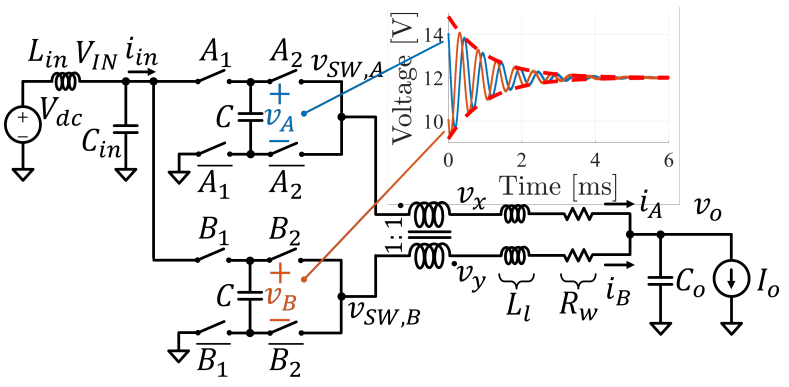

Figure 1: Schematic of two-phase, three-level FCML converter with tightly coupled inductors. The flying capacitor natural balancing with estimated envelope is plotted. An input filter is included to support the disturbance analysis.

Flying capacitor balancing is critical to avoid increased current ripple and switch stress in FCML converters [5-7]. While FCML converters exhibit natural balancing that guarantees the initial capacitor voltage imbalances will eventually decay, it is challenging to analytically predict how long the process takes. Moreover, natural balancing cannot correct for periodic disturbances that result in large capacitor voltage imbalances at steady-state [8].

This paper investigates voltage balancing of flying capacitors in the multiphase FCML converter with coupled inductors. The coupled inductor FCML converter intrinsically reduces the steady-state capacitor voltage imbalance. The steady-state imbalances caused by source impedances and switching delays are significantly reduced. The natural balancing dynamics of the converter are derived and verified with simulations and experiments.

\section{Natural Balancing of FCML Converter with Coupled Inductors}

To highlight the key principles, We assume strong coupling between the inductors and thus a negligible $L_{\mu}$. Therefore, the two phases have the same current $i_{A}=i_{B}$. The flying capacitors $C_{A}=C_{B}=C$ are symmetric and both phases have the same leakage inductance $L_{l}$ and winding resistance $R_{w}$. As explained in [5], if the inductors are not coupled, the converter exhibits natural balancing that balances them to a steady-state equilibrium if there is an initial imbalance. With a coupled inductor, the flying capacitors oscillate with each other, as shown in Fig. 1. To analyze the benefits of including a coupled inductor for balancing a multiphase FCML converter, we focus on the oscillatory behavior of the flying capacitor voltages, $\tilde{v}_{A}$ and $\tilde{v}_{B}$, where the total voltages are $v_{A}=\frac{V_{d c}}{2}+\tilde{v}_{A}$ and 
$v_{B}=\frac{V_{d c}}{2}+\tilde{v}_{B}$. The dc input and output pertain to steady-state operation and are neglected in this analysis.

The switching waveforms for $0<d \leq 0.25$ are shown in Fig. 2 .

For the natural balancing analysis, we assume no periodic disturbances so $\Delta V_{1}=\Delta V_{2}=\Delta d=0$. The period $T$ is divided into eight sub-periods, one for each set of switch positions. We solve the circuit in each sub-period to find a dynamic model of natural balancing. Only three unique sub-circuits need to be solved for all possible sub-periods, depending on if there are zero, one, or two flying capacitors connected to the output. This method may be applied to converters with many phases and levels. In sub-period \#1, switch $A_{1}$ connects capacitor $\mathrm{A}$ and the converter has the equations

$$
\begin{gathered}
\tilde{i}_{A}=C \frac{d \tilde{v}_{A}}{d t}, \\
\frac{\tilde{v}_{A}}{2}-L_{l} \frac{d \tilde{i}_{A}}{d t}-R_{w} \tilde{i}_{A}=0 .
\end{gathered}
$$

From (1) and (2), the solution for sub-period \#1 is

$$
\begin{aligned}
{\left[\begin{array}{c}
\tilde{v}_{A}(d T) \\
\tilde{v}_{B}(d T) \\
\tilde{i}_{A}(d T)
\end{array}\right] } & =\underbrace{\left[\begin{array}{ccc}
\alpha(d T) & 0 & \beta(d T) \\
0 & 1 & 0 \\
C \alpha^{\prime}(d T) & 0 & C \beta^{\prime}(d T)
\end{array}\right]}_{\mathbf{T}_{1}}\left[\begin{array}{c}
\tilde{v}_{A}(0) \\
\tilde{v}_{B}(0) \\
\tilde{i}_{A}(0)
\end{array}\right], \\
\alpha(t) & =e^{-\frac{R_{w}}{2 L_{l}} t}\left[\cos \left(\omega_{d} t\right)+\frac{R_{w}}{2 L_{l} \omega_{d}} \sin \left(\omega_{d} t\right)\right], \\
\beta(t) & =e^{-\frac{R_{w}}{2 L_{l}} t} \frac{1}{C \omega_{d}} \sin \left(\omega_{d} t\right),
\end{aligned}
$$

where $\omega_{d}=\frac{1}{2} \sqrt{\frac{2}{C L_{l}}-\left(\frac{R_{w}}{L_{l}}\right)^{2}} . \quad \mathbf{T}_{\mathbf{1}}$ updates the state variables through sub-period \#1. The odd-numbered sub-periods also have

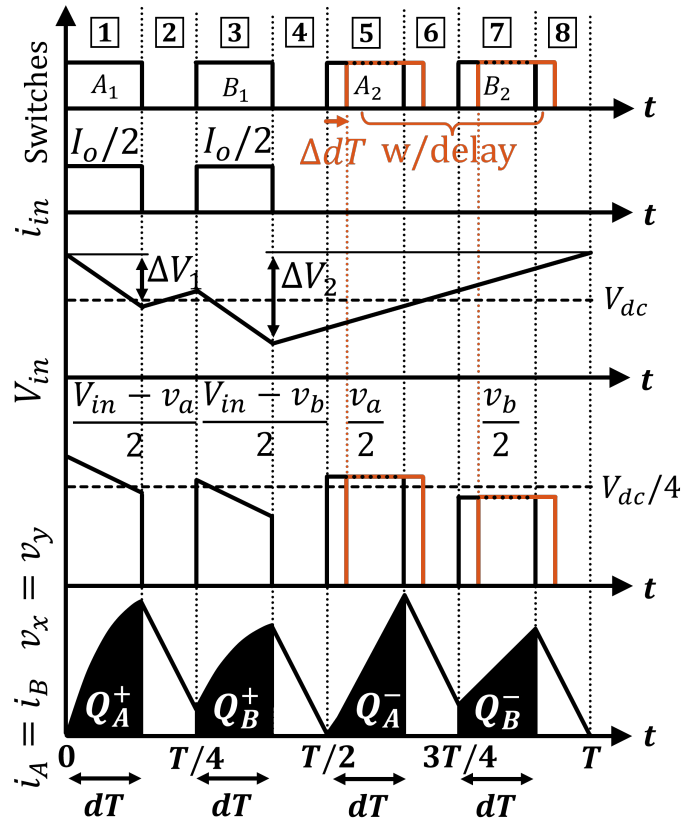

Figure 2: Switching waveforms for $0<d \leq 0.25$. The period $T$ is divided into eight sub-periods, with one for each set of switch positions. $V_{i n}$ varies if there is a source impedance and the $A_{2}$ and $B_{2}$ switches are delayed by $\Delta d$ if there is a switching delay.

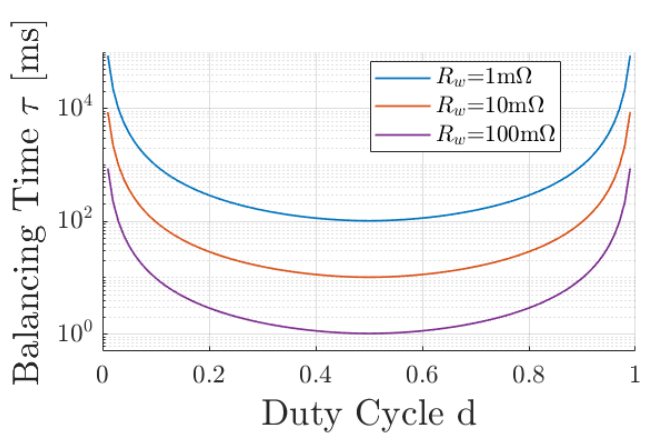

Figure 3: Predicted natural balancing time plotted against duty cycle $d$ and winding resistance $R_{w}$. one capacitor connected and we can reuse the solution in $(3)$ - (5) for $\tilde{v}_{B}$ or change the sign of the capacitor connection. The even-numbered sub-periods have no capacitor connected and only the inductor current changes by decaying through $R_{w}$. Combining the state update matrices $\mathbf{T}_{\mathbf{1}}$ through $\mathbf{T}_{\mathbf{8}}$, we find the discrete update equation

$$
\mathbf{x}(t+T)=\mathbf{T}_{\mathbf{8}} \mathbf{T}_{\mathbf{7}} \mathbf{T}_{\mathbf{6}} \mathbf{T}_{\mathbf{5}} \mathbf{T}_{\mathbf{4}} \mathbf{T}_{\mathbf{3}} \mathbf{T}_{\mathbf{2}} \mathbf{T}_{\mathbf{1}} \mathbf{x}(t)=\mathbf{T}_{\text {cycle }} \mathbf{x}(t)
$$

where the state variables are $\mathbf{x}=\left[\begin{array}{lll}\tilde{v}_{A} & \tilde{v}_{B} & \tilde{i}_{L}\end{array}\right]^{T}$. For the other duty cycle regimes in Table 1 , we can find $\mathbf{T}_{\mathbf{c y c l e}}$ by solving different sub-circuits; only three unique solutions like (3) -(5) are need to solve for natural balancing with any duty cycle. Using the central approximation of the derivative, (6) is converted to

$$
\dot{\mathbf{x}}(t) \approx \frac{\mathbf{x}(t+T)-\mathbf{x}(t-T)}{2 T}=\frac{\mathbf{T}_{\text {cycle }}-\mathbf{T}_{\text {cycle }}{ }^{-1}}{2 T} \mathbf{x}(t)=\mathbf{A} \mathbf{x}(t),
$$

an approximate continuous-time dynamic system. We estimate the natural balancing time from the eigenvalues of the state matrix A. As Fig. 1 suggests, there are a pair of complex conjugate eigenvalues for the oscillatory 
behavior. For example, the eigenvalues with the circuit parameters in Table $2, R_{w}=100 \mathrm{~m} \Omega$, and $d=0.5$ are $\lambda_{1}=-4.68 \times 10^{5}, \lambda_{2,3}=-970 \pm 1.28 \times 10^{4} j$. The real eigenvalue decays rapidly and we can estimate the time constant of natural balancing plotted on Fig. 1 as $\tau=-\frac{1}{\operatorname{Re}\left(\lambda_{2,3}\right)}=1.03 \mathrm{~ms}$. The envelope provides a good estimate of the flying capacitor balancing time without needing to find the oscillatory behavior. Figure 3 shows the predicted balancing time for different winding resistances $R_{w}$ across the duty cycle range. The balancing time is smaller the longer the capacitors are connected to the output during a period and the minimum balancing time occurs at $d=0.5$. The balancing time is symmetric as the capacitor connection time is also symmetric about $d=0.5$.

\section{Steady-State Balancing of FCML Converter with Coupled Inductors}

Figure 1 includes a source impedance that causes a steady-state capacitor voltage imbalance which the capacitors settle to after natural balancing [8]. To derive the steady-state imbalance, we assume that the flying capacitors are large so their voltages are constant in a switching period and that $R_{w}$ is negligible. Since $R_{w}=0$, natural balancing does not play a role in the proceeding analysis and the steady-state equilibrium is intrinsic to the coupled inductor.

\subsection{Derivation of Steady-State Imbalance Due to Source Impedance}

Since the currents over the leakage inductances are the same, $v_{x}=v_{y}=\frac{v_{S W, A}+v_{S W, B}}{2}$ by inspection of Fig. 1 . If the input inductance $L_{i n}$ is large and the current ripple of $i_{i n}$ is small, the two branches can be modeled as current sources such that $V_{i n}$ varies linearly depending on $i_{i n}$, as shown in Fig. 2 . In sub-periods \#1 and \#3, one phase draws $i_{i n}=I_{o} / 2$, causing $V_{i n}$ to decrease by $\Delta V_{1}=\frac{I_{o} T}{C}\left(\frac{d}{2}-d^{2}\right)$. In sub-periods $\# 4$ through $\# 8, V_{i n}$ increases by $\Delta V_{2}=\frac{I_{o} T}{C}\left(\frac{d}{2}+d^{2}\right)$. The average $V_{i n}$ is greater than $V_{d c}$ in sub-period \#1 and less than $V_{d c}$ in sub-period \#3.

In steady-state, the inductor current and flying capacitor voltages are constant. The steady-state capacitor voltage imbalances must therefore satisfy three conditions: $i_{A}(0)=i_{A}(T), Q_{A}^{+}=Q_{A}^{-}$, and $Q_{B}^{+}=Q_{B}^{-}$. The inductor current starts and ends at the same value in a period if $v_{o}=d V_{d c}$, since the average voltage of $V_{i n}$ is still $V_{d c}$. To satisfy the charge balance conditions, we first solve for the inductor current in every sub-period. In sub-period \#1, switch $A_{1}$ is on, $V_{i n}^{\# 1}(t)=V_{d c}+\frac{\Delta V_{2}}{2}-\frac{\Delta V_{1} t}{d T}$, and the inductor current is

$$
i_{A}^{\# 1}(t) d t=\frac{1}{L} \int\left(\frac{V_{i n}^{\# 1}(t)-v_{a}}{2}-v_{o}\right) d t=\frac{1}{L}\left[\frac{\Delta V_{1} t^{2}}{4 d T}+\left(\frac{v_{a}}{2}-\frac{V_{d c}}{2}+v_{o}-\frac{\Delta V_{2}}{4}\right) t\right],
$$

where we ignore the dc value of current because it will charge and discharge the capacitors equally. The charge transferred into capacitor A during sub-period 1 is the integral of the current:

$$
Q_{A}^{+}=\int_{0}^{d T} i_{A}^{\# 1}(t) d t=-\frac{d^{2} T^{2}}{24 L}\left[6 v_{a}-6 V_{d c}+12 v_{o}+2 \Delta V_{1}-3 \Delta V_{2}\right]
$$

Solving for the current in sub-periods \#2 through \#8, we similarly find $Q_{A}^{-}, Q_{B}^{+}$, and $Q_{B}^{-}$. If we substitute $v_{o}=d V_{d c}$ and invoke the steady-state conditions $Q_{A}^{+}=Q_{A}^{-}$and $Q_{B}^{+}=Q_{B}^{-}$, the capacitor voltage imbalances $\tilde{v_{A}}$ and $\tilde{v_{B}}$ are

$$
\left.\tilde{v_{A}}\right|_{Q_{A}^{+}=Q_{A}^{-}}=-\left.\tilde{v_{B}}\right|_{Q_{B}^{+}=Q_{B}^{-}}=\frac{I_{o} T}{48 C}\left(-4 d^{2}+5 d\right)
$$

to satisfy the conditions where the capacitor voltages are constant. This result is derived assuming the winding resistance $R_{w}$ is zero. Therefore, this steady-state imbalance is not dependent on natural balancing; rather, it is a fundamental property of the coupled inductor FCML converter. The coupled inductor makes both phases share the 
same current ripple and switch node $v_{x}=v_{y}$, which facilitates a steady-state equilibrium of the capacitor voltages. The capacitor A imbalance is positive and the capacitor B imbalance is negative, which counteracts $V_{i n}>V_{d c}$ in sub-period \#1 and $V_{i n}<V_{d c}$ in sub-period \#3. The magnitudes of the imbalances ensure $Q_{A}^{+}=Q_{A}^{-}$and $Q_{B}^{+}=Q_{B}^{-}$.

In a converter with uncoupled induc-

Table 1: Coefficients of Steady-State Capacitor Voltage Imbalance tors and $R_{w}=0$, the charge transferred into the capacitors in one period are

$$
\begin{aligned}
\Delta Q_{A}^{*} & =-\frac{d T^{2}}{48 L_{l} C_{i n}}\left(4 I_{o} d^{3} T+I_{o} d^{2} T\right), \\
\Delta Q_{B}^{*} & =\frac{d T^{2}}{48 L_{l} C_{i n}}\left(-4 I_{o} d^{3} T+5 I_{o} d^{2} T\right),
\end{aligned}
$$

\begin{tabular}{ccc}
\hline Duty Cycle Range & $\gamma$ & $\boldsymbol{\rho}$ \\
\hline $0<d \leq 0.25$ & $\frac{1}{48}\left(-4 d^{2}+5 d\right)$ & $2 \Delta d$ \\
\hline $0.25<d \leq 0.5$ & $\frac{1}{96}\left(8 d^{2}+2 d+1\right)$ & $-\frac{16 d^{2} \Delta d-8 d \Delta d+2 \Delta d}{8 d^{2}-8 d+8 \Delta d^{2}+1}$ \\
\hline $0.5<d \leq 0.75$ & $\frac{1}{96} \frac{-64 d^{4}+48 d^{2}-86 d+21}{8 d^{2}-8 d+1}$ & $-\frac{16 d^{2} \Delta d-24 d \Delta d+10 \Delta d}{8 d^{2}-8 d+8 \Delta d^{2}+1}$ \\
\hline $0.75<d<1$ & $\frac{1}{48}\left(4 d^{2}+5 d-3\right)$ & $2 \Delta d$ \\
\hline
\end{tabular}

which are never zero, so the capacitor voltages diverge. Only natural balancing stabilizes the capacitor voltages [8]. However, natural balancing is unpredictable and is weakened by minimizing the winding resistance. The coupled inductor self-balancing is separate from and stronger than natural balancing. If we repeat the preceding analysis for the other duty cycle regimes in Table 1, the steady-state capacitor voltage imbalances are

$$
\left.\tilde{v_{A}}\right|_{\text {steady-state }}=-\left.\tilde{v_{B}}\right|_{\text {steady-state }}=\gamma \frac{I_{o} T}{C_{\text {in }}},
$$

where $\gamma$ is a dimensionless coefficient that depends on the duty cycle, as listed in Table 1 . The capacitor voltage imbalance scales with variations in $V_{i n}$ and thus scales with $I_{o}$ and $T$ and inversely with $C_{i n}$.

\subsection{Derivation of Steady-State Imbalance Due To Switching Delay}

Switching delays arising from rise and fall times or signal propagation are another periodic disturbance that cause steady-state capacitor voltage imbalances [8]. Fig 2 shows the second set of switches $A_{2}$ and $B_{2}$ of both phases being delayed by $\Delta d T$. Since $V_{i n}$ is now constant, it is simpler to find the steady-state imbalances that satisfy $Q_{A}^{+}=Q_{A}^{-}$and $Q_{B}^{+}=Q_{B}^{-}$. For example, charge entering capacitor $\mathrm{A}$ is the area under the current in sub-period 1:

$$
Q_{A}^{+}=\frac{1}{2} d T \frac{1}{L}\left(\frac{V_{d c}-v_{A}}{2}-v_{o}\right) d T=\frac{(d T)^{2}\left(V_{d c}-v_{A}-2 v_{o}\right)}{4 L},
$$

Following the procedure in section 3.1, we find the current in sub-periods \#2-\#8 and the charges $Q_{B}^{+}, Q_{A}^{-}$, and $Q_{B}^{-}$. If we invoke the steady-state condition $Q_{A}^{+}=Q_{A}^{-}$and $Q_{B}^{+}=Q_{B}^{-}$, the steady-state capacitor voltage imbalances are

$$
\left.\tilde{v_{A}}\right|_{\text {steady-state, delay }}=-\left.\tilde{v_{B}}\right|_{\text {steady-state, delay }}=\rho V_{d c}
$$

where $\rho$ is listed in Table 1 for the different duty cycle regimes. The self-balancing mechanism is not dependent on natural balancing, unlike a converter with uncoupled inductors.

\section{Experimental and Simulation Verifications of Steady-State Imbalance}

Figure 4 shows simulation and experimental verifications of the predicted steady-state imbalances with a two-phase, three-level FCML con-
Table 2: Circuit Parameters for Simulation and Experiments

\begin{tabular}{ccccccc}
\hline$f_{s w}$ & $V_{d c}$ & $C$ & $L_{l}$ & $L_{\mu}$ & $I_{o}$ & $C_{i n}$ \\
\hline $1 \mathrm{MHz}$ & $24 \mathrm{~V}$ & $22 \mu \mathrm{F}$ & $220 \mathrm{nH}$ & $4.48 \mu \mathrm{H}$ & $5 \mathrm{~A}$ & $22 \mu \mathrm{F}$ \\
\hline
\end{tabular}


verter using the circuit parameters in Table 2.

The input inductance $L_{i n}$ and winding resistance $R_{w}$ are parasitic in the experimental setup and the simulations use $L_{i n}=100 \mathrm{nH}$ and $R_{w}=10 \mathrm{~m} \Omega$. Figure 4 a shows the simulated imbalance matches the predicted values well, with small deviations due to current in the magnetizing inductance that was assumed to be negligible. Moreover, the capacitor voltage imbalances are 10 to 100 times lower in a converter with uncoupled inductors equal to the leakage inductance $L_{l}=220 \mathrm{nH}$. The coupled inductor self-balancing mechanism is much stronger than natural balancing and $R_{w}$ may be minimized to improve efficiency without affecting the balancing.

Fig. 4b shows experimental flying capacitor voltages of a twophase, three-level FCML converter with the duty cycle propagation delay $\Delta d$ varying between -0.08 and +0.08 . The measured capacitor voltages agree with theory with a coupled inductor, with some deviations due to some asymmetry in the experimental setup. The imbalances are lower with a coupled inductor than with a coupled inductor. Extended experimental results will be presented in the final paper.

\section{Conclusion}

This paper investigates flying capacitor voltage balancing in the multiphase FCML converter with coupled inductors. The coupled inductor intrinsically reduces the steady-state imbalance due to periodic disturbances compared to converters with uncoupled inductors. The steady-state imbalance induced by source impedances and switching delays are predicted and are shown to be independent of natural balancing. Natural balancing of the flying capacitors is analyzed and shown to be oscillatory due to the coupled inductor. A dynamic model of natural balancing and the balancing time is

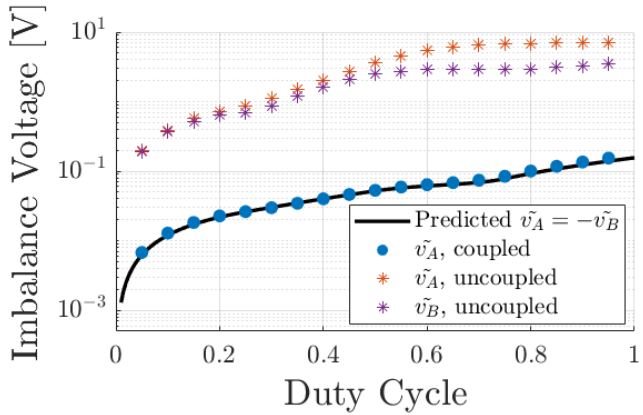

(a)

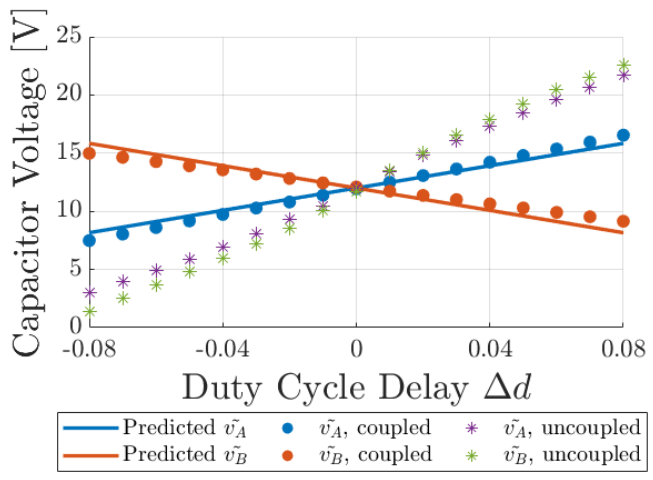

(b)

Figure 4: (a) Comparison of predicted and simulated capacitor voltage imbalances for coupled and uncoupled inductors. (b) Measured capacitor voltage imbalances for varying duty cycle delay with coupled and uncoupled inductors.

estimated. The predicted steady-state and natural balancing behavior is verified with simulations and experiments.

\section{References}

[1] X. Zhou, P.-L. Wong, P. Xu, F. C. Lee, and A. Q. Huang, "Investigation of Candidate VRM Topologies for Future Microprocessors," IEEE Transactions on Power Electronics, vol. 15, no. 6, pp. 1172-1182, Nov. 2000.

[2] J. Rodriguez et al., "Multilevel Converters: An Enabling Technology for High-Power Applications," Proceedings of the IEEE, vol. 97, no. 11, pp. 1786-1817, Nov. 2009.

[3] P.-L. Wong, "Performance Improvements of Multi-Channel Interleaving Voltage Regulator Modules with Integrated Coupling Inductors," PhD Thesis, Virginia Tech, Blacksburg, VA, 2001.

[4] D. Zhou, Y. Elasser, J. Baek, C. R. Sullivan and M. Chen, "Inductance Dual Model and Control of Multiphase Coupled Inductor Buck Converter," IEEE Workshop on Control and Modeling for Power Electronics, 2020, pp. 1-8.

[5] B. P. McGrath and D. G. Holmes, "Analytical Modelling of Voltage Balance Dynamics for a Flying Capacitor Multilevel Converter," IEEE Power Electronics Specialists Conference, 2007, pp. 1810-1816.

[6] S. Thielemans et al., "Improved Natural Balancing With Modified Phase-Shifted PWM for Single-Leg Five-Level Flying-Capacitor Converters," IEEE Transactions on Power Electronics, vol. 27, no. 4, pp. 1658-1667, April 2012.

[7] Z. Xia, B. L. Dobbins, J. T. Stauth, "Natural Balancing of Flying Capacitor Multilevel Converters at Nominal Conversion Ratios", IEEE Control and Modeling for Power Electronics, pp. 1-8, 2019.

[8] Z. Ye, Y. Lei, Z. Liao, and R. C. N. Pilawa-Podgurski, "Investigation of Capacitor Voltage Balancing in Practical Implementations of Flying Capacitor Multilevel Converters," IEEE Workshop on Control and Modeling for Power Electronics, 2017, pp. 1-7. 